\title{
A strange case of foreign body embolism in the right lower pulmonary artery
}

\author{
Angelo Paolo Ciarrocchi ${ }^{1}$ (1) - Anna Mariantonia Parisi ${ }^{1}$. Alessio Campisi ${ }^{1} \cdot$ Sara Mazzarra $^{1} \cdot$ Desideria Argnani $^{1}$. \\ Stefano Congiu ${ }^{1} \cdot$ Stefano Sanna ${ }^{1} \cdot$ Franco Stella $^{1}$
}

Received: 7 September 2020 / Accepted: 12 December 2020 / Published online: 5 January 2021

(c) The Japanese Association for Thoracic Surgery 2021

\begin{abstract}
Foreign bodies in the pulmonary circulation have been documented in the literature, with almost all cases being iatrogenic, involving venous catheters, or due to penetrating foreign body emboli. Foreign body pulmonary emboli are often difficult to diagnose due to their varied clinical presentation, the nature of the embolizing material and dubious radiological features. We describe the case of a patient who experienced episodes of massive hemoptysis with inconclusive radiological findings, who underwent a thoracotomy with the discovery of a wooden object of $7 \mathrm{~cm}$ in length in the right lower lobe artery, with no apparent mechanism of injury.
\end{abstract}

Keywords Pulmonary artery $\cdot$ Foreign body $\cdot$ Nonthrombotic pulmonary embolism

\section{Introduction}

Foreign bodies in the pulmonary circulation have been documented in the literature, with almost all cases being iatrogenic, usually due to the fracture and embolism of central or peripheral venous catheters. The remaining cases described involve traumatic emboli of foreign bodies following explosions, bullet wounds or from flying particles in industrial accidents [1-3]. Foreign body pulmonary emboli are often difficult to diagnose due to their varied clinical presentation, the nature of the embolizing material and dubious radiological features. An accurate diagnosis requires expert knowledge of the diverse radiological findings. Non-symptomatic cases can undergo an observation period, however, most patients eventually develop symptoms, either due to

Angelo Paolo Ciarrocchi and Anna Mariantonia Parisi have equally contributed to the article.

Supplementary Information The online version contains supplementary material available at https://doi.org/10.1007/s1174 8-020-01570-5.

Angelo Paolo Ciarrocchi

angelo.ciarrocchi@studio.unibo.it

1 Thoracic Surgery Unit, Department of Thoracic Diseases, G.B. Morgagni-L. Pierantoni Hospital, University of Bologna, 34 Carlo Forlanini Street, 47121 Forli, Italy contamination or as a result of continuous embolization [2]. The primary curative approach in cases involving a foreign object in the proximal pulmonary arteries is endovascular. In case of a failure of a primary approach or an undiagnosed foreign body in the pulmonary artery, surgery is often performed [4]. To the best of our knowledge, no cases of noniatrogenic pulmonary artery foreign body have been reported in a patient without a history of penetrating trauma.

\section{Case presentation}

A 46-year-old male presented to our emergency department after repeated episodes of massive haemoptysis after coughing bouts. The patient was admitted to the internal medicine ward and underwent a chest X-ray, which revealed a right basal opacity. He underwent a chest CT scan with the finding of an endoluminal filling defect of the artery of the lateral basal segment of the right lower lobe with uneven contrast enhancement, dilation of the vessel and the presence of large rings of perivascular ground glass, most likely due to alveolar hemorrhage (Fig. 1). According to the radiological report, these findings were compatible with pulmonary angiosarcoma or neoplastic thrombi. A flexible bronchoscopy revealed a small quantity of blood and small blood clots in the right bronchi, in particular in the right lower bronchus, without 


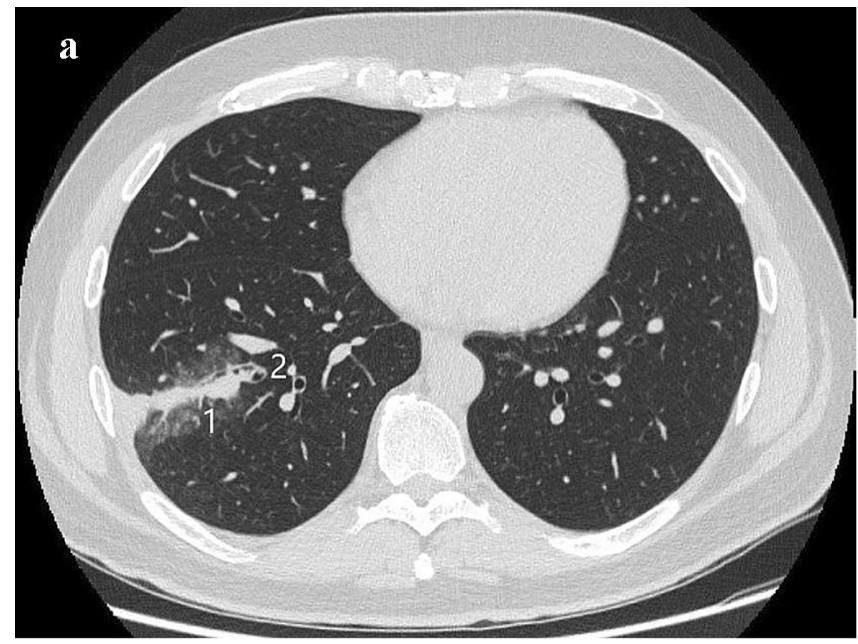

Fig. 1 a Chest CT scan showing diffuse areas of alveolar hemorrhage in the right lower lobe. (1) Perivascular ground-glass opacity. (2) Lateral basal segment bronchus. b An endoluminal filling defect of the

any other alterations of the trachea or bronchi. The patient was subsequently referred to our unit where we performed a cranial CT scan which was negative and an FDG-PET scan which revealed a weak tracer accumulation (SUV$\max 1.9$ ) at the level of the suspected alveolar hemorrhage of the lower lobe. After a multidisciplinary discussion, it was decided to perform an intraoperative biopsy of the affected artery via thoracotomy. Upon opening, the lower lobe was found to be necrotic, most likely due to multiple and continuous infectious emboli. A nodule of approximately $1.2 \mathrm{~cm}$ attached to the artery of the lateral basal segment was sent for intraoperative histological analysis and was found to be lung parenchyma with lymphoplasmacytic infiltrates and areas of necrosis, fibrin

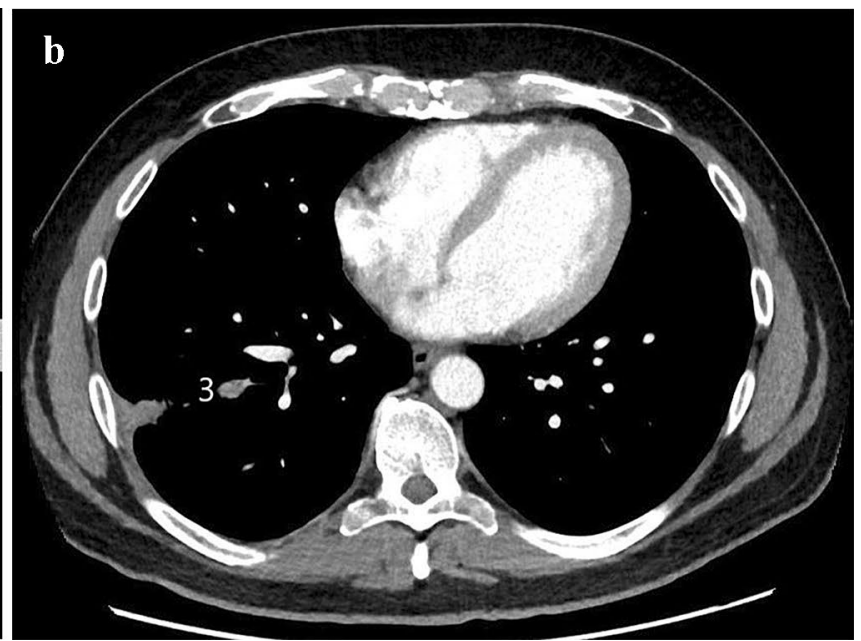

artery of the lateral basal segment of the right lower lobe. (3) Lateral basal segment artery

and acute inflammation. The right lower lobe artery was isolated in the interlobar fissure, at which point a cannulalike object was palpated inside the artery. The artery was clamped proximally and distally of the mass using two hemostatic clamps. The artery was dissected longitudinally upon which a linear tubular wooden object of $7 \mathrm{~cm}$ in length was found inside the artery lumen and removed (Fig. 2). The artery was sutured with a continuous suture of Prolene 6-0 (Ethicon Inc, Piscataway, NJ). The patient experienced a slight fever $\left(37.8^{\circ} \mathrm{C}\right)$ on post-operative day 4 which resolved spontaneously. On postoperative day 6 , the chest tubes were removed and the patient was discharged the next day without further complications. At the 1 month and 3 month follow-ups, the patient was in
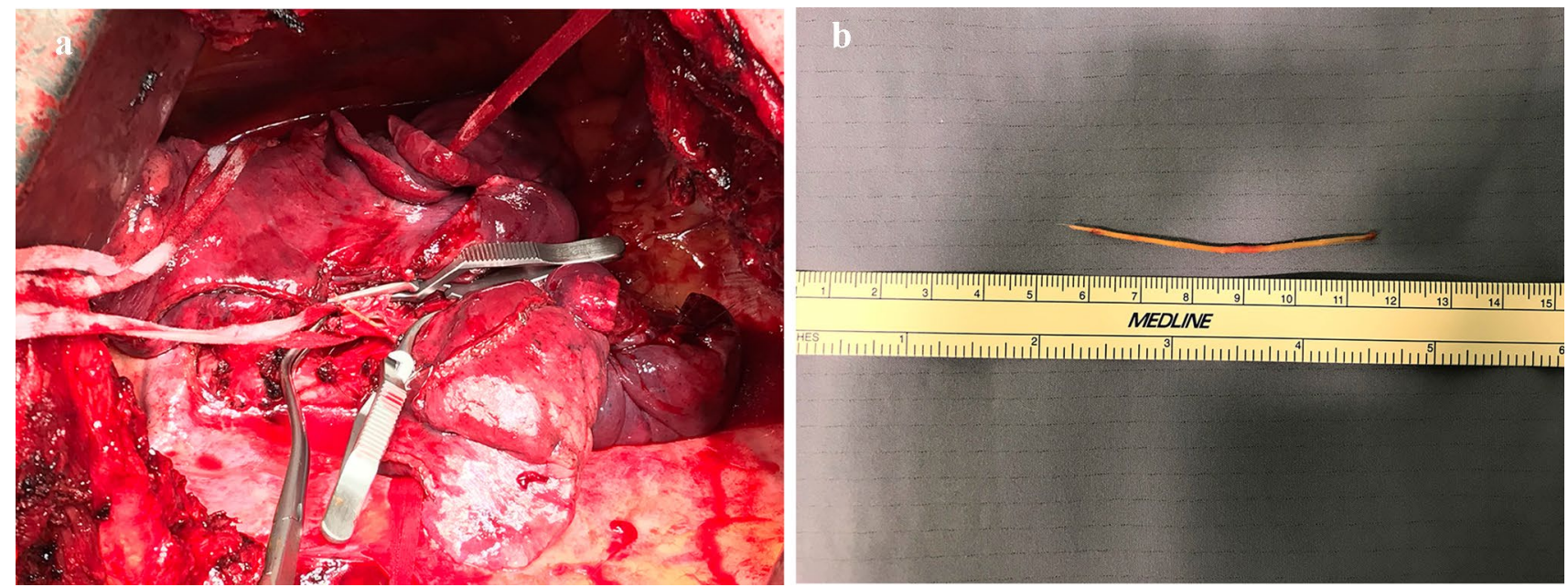

Fig. 2 a The dissected artery of the lateral basal segment of the right lower lobe with the wooden foreign object protruding from it. b The wooden object measuring approximately $7 \mathrm{~cm}$ after being removed from the artery 
excellent medical condition, without additional therapy and no limitations to his daily life. Additional follow-up will be performed telephonically.

\section{Discussion}

The majority of reported cases of foreign bodies in the pulmonary vasculature are iatrogenic, involving fragmented catheter material and intravascular devices [1]. The remainder of the cases involve emboli following penetrating injury from gunshot wounds or shrapnel wounds after explosions or industrial accidents [2]. An agreed definitive management strategy for the removal of a pulmonary artery foreign body does not exist, however, when feasible the initial curative approach is most often an attempted endovascular retrieval, which, if unsuccessful, is followed by surgery [4]. The general consensus is that foreign bodies must be urgently removed if the patient is young or the foreign body is $>5 \mathrm{~mm}$ in diameter or $10 \mathrm{~cm}$ in length, the tissues surrounding the object are heavily damaged and or the area is contaminated [5]. A preoperative diagnosis could not be made and it was decided to perform an intraoperative biopsy due to the radiological suspicion of an angiosarcoma. Our patient had no history of penetrating trauma and no known mechanism of injury. After postoperative consultations, an image of the foreign object was shown to the patient, who identified it as a pine needle from a Maritime Pine tree (Pinus pinaster) which surrounds his home in the countryside. According to the patient, during the months of SARS-COV-2 lockdown he had run approximately $300 \mathrm{~km}$ in the countryside surrounding his house and believes he could have easily inhaled a pine needle. The right lower lobe presented extensive necrosis and inflammation, most likely due to continuous infectious emboli from the wooden foreign body, which further highlights the need to remove foreign bodies in the pulmonary vasculature when symptomatic.

\section{Conclusions}

Foreign objects in the pulmonary vasculature are not rare and mostly due to iatrogenic causes or after exploding or penetrating trauma. No cases exist in the literature of such an event without a known mechanism of injury. Due to the complicated nature of this case, a thoracotomy was performed to determine the nature of the unidentified mass present in the artery. Fortunately, the foreign object could be located and removed as it presented a risk for further lung necrosis from multiple recurrent infectious emboli.

Funding No funding used.

\section{Compliance with ethical standards}

Conflict of interest No conflict of interest to declare.

Informed consent Written informed consent was obtained from the patient for publication of this manuscript and any accompanying images or videos.

\section{References}

1. Dell'Amore A, Ammari C, Campisi A, D'Andrea R. Peripheral venous catheter fracture with embolism into the pulmonary artery. J Thorac Dis. 2016;8(12):E1581-4.

2. Sakai T, Uehara H, Takahashi S, Yamamoto M, Kawamura M, Kondo H. Surgical removal of a foreign body in the pulmonary artery. Trauma. 2019;21(2):152-4.

3. Boomer LA, Watkins DJ, O'Donovan J, Kenney BD, Yates AR, Besner GE. An unusual case of foreign body pulmonary embolus: case report and review of penetrating trauma at a pediatric trauma center. Pediatr Surg Int. 2015;31(3):241-7.

4. Schechter MA, O'Brien PJ, Cox MW. Retrieval of iatrogenic intravascular foreign bodies. J Vasc Surg. 2013;57(1):276-81.

5. Lu K, Gandhi S, Qureshi MA, Wright AS, Kantathut N, Noeller TP. Approach to management of intravascular missile emboli: review of the literature and case report. West J Emerg Med. 2015;16(4):489-96.

Publisher's Note Springer Nature remains neutral with regard to jurisdictional claims in published maps and institutional affiliations. 